\title{
Reusable SERS active substrates for ultrasensitive molecular detection
}

Hardeep Singh Gill ${ }^{1,2}$, Sammaiah Thota ${ }^{1}$, Lian Li $^{3}$, Haizhou Ren ${ }^{2}$, Ravi Mosurkal ${ }^{3, a)}$, and Jayant Kumar ${ }^{1,2, a)}$

\footnotetext{
${ }^{I}$ Center for Advanced Materials, University of Massachusetts Lowell, Lowell, MA 01854, USA

${ }^{2}$ Department of Physics \& Applied Physics, University of Massachusetts Lowell, Lowell, MA 01854, USA

${ }^{3}$ US Army Natick Soldier Research, Development and Engineering Center, Natick, MA 01760, USA
}

\begin{abstract}
Silver nanoparticles coated silicon nanospikes were investigated as efficient and reusable SERS active substrates. Femtosecond laser pulses fabricated silicon nanospikes were made SERS active through deposition of silver nanoparticles by reducing silver nitrate. The prepared substrates offer good SERS reproducibility with high enhancement factors. The SERS detection limits of 1 pico-molar from Rhodamine 6G and 100 pico-molar from Methylene blue were demonstrated. In addition, these SERS substrates are reusable by simple washing and no observable degradation of sensitivity was measured.
\end{abstract}

Keywords: SERS; Femtosecond; Nanostructures; Detectors

\section{Introduction}

Surface-enhanced Raman spectroscopy (SERS) is a powerful analytical tool for ultra-sensitive and selective detection of chemicals using nanostructured metallic materials. ${ }^{1-3}$ Numerous research efforts have been devoted to develop SERS active substrates using facile and cost-effective fabrication approaches. ${ }^{4-5}$ Among all the SERS active substrates, gold and silver based nanostructures with sharp edges ("hot spots") are commonly utilized due to their high sensitivity and good biocompatibility. ${ }^{6,7}$ Single molecule detection with the SERS substrates has been demonstrated. ${ }^{8}$ However, most of the approaches used so far are characterized by major challenges in stability and reproducibility of the Raman signal. ${ }^{9,10}$ The 'hot-spots' on those substrates are randomly distributed and consequently the SERS responses often vary from spot to spot over the substrate surface. ${ }^{11,12}$ Recently, femtosecond (FS) laser machined silicon nanospike structures and their replicas were utilized as viable SERS substrates. ${ }^{13}$, ${ }^{14}$ These patterned nanostructures prepared using FS-laser in conjunction with coating of metallic layers

\footnotetext{
a)Electronic mail: Jayant Kumar@uml.edu. and Ravi.Mosurkal.civ@mail.mil
} 
by evaporation, ${ }^{14}$ and chemical plating, ${ }^{15}$ yielded an enhancement factor (EF) of $10^{7}$. However, the practical use of these fabricated substrates was frequently limited by instability and poor reproducibility of the Raman signals. ${ }^{15,16}$ The lack of uniformity and persistent performance might be attributed to randomly distributed "hot spots" on the nanostructures. Moreover, it has been reported that the SERS substrates in general are lack of reproducibility for the Raman measurements and could not be reused. ${ }^{17}$ The absence of reproducibility and reusability has restricted these substrates as cost-effective SERS sensors for practical applications. In this communication, we report an efficient approach to fabricate uniform, large area, ultrasensitive and reusable SERS substrates that can be repeatedly used in Raman experiments without losing their sensitivity. These prepared substrates provide good SERS reproducibility with high enhancement factors, enabling the ultra-sensitive detection of 1 picomolar Rhodamine6G (R6G) and 100 pico-molar Methylene blue (MB) analytes adsorbed from aqueous solutions. These substrates were reusable and no significant change was observed in the enhancement factor upon a number of sensing and cleaning cycles.

\section{Experimental details}

Silicon wafers were first cleaned with acetone to remove any possible contaminants and were then rinsed with water, and dried with nitrogen gas. An FS Ti:sapphire laser operating at $800 \mathrm{~nm}$ (100 FS, 1 $\mathrm{kHz}$ repetition rate and $1 \mathrm{~W}$ average power) was utilized to produce the nanostructures on the silicon wafer. The cleaned substrates were subjected to the focused FS laser beam at $400 \mathrm{~nm}$ (frequencydoubled) in methanol on a computer controlled X-Y stage. The nanospike structures with an area of 0.5 $\mathrm{cm} \times 0.5 \mathrm{~cm}$ were fabricated. R6G, MB, silver nitrate, hydrazine monohydrate, Vitamin $\mathrm{C}$, and citric acid were purchased from Sigma-Aldrich and used without further purification. All solutions were prepared with distilled water. The laser machined silicon nanostructures were first rinsed with distilled water. The cleaned nanostructured substrates were then dipped into the aqueous solution of silver nitrate (100 mM) for $30 \mathrm{~min}$ and followed by addition of several drops of aqueous hydrazine hydrate $(3 \mathrm{M})$ onto the substrates. The treated silicon nanostructured substrate was then heated at $50{ }^{\circ} \mathrm{C}$ in a Vitamin $\mathrm{C}$ (0.3 M): citric acid (2.6 mM) aqueous solution (6:1) for 1 hour. The substrate was then rinsed with distilled water and dried with nitrogen gas. For comparison, a silicon wafer without the nanostructures was also coated with silver nanoparticles by the same reduction method. The nanospikes and the silver nanoparticles coated nanostructure were examined by scanning electron microscopy (SEM). Figure 1 shows the SEM images of nanospike structures with and without silver nanoparticles, and the silver nanoparticles coated flat silicon surface. As shown in Figure 1 (a), the nanospikes with height and 
spacing of 200-300 nm were fabricated by the FS laser pulses. Silver nanoparticles with particle size about $50 \mathrm{~nm}$ were uniformly formed on the silicon nanostructures (Figure 1 (b)). Figure 1(c) shows the SEM image of the silver nanoparticles coated flat silicon surface. Fewer amounts of silver nanoparticles were loaded on the flat Si surface as compared to that of the nanostructured one.

R6G and MB (commonly used test analytes) solutions with concentrations of $10^{-5} \mathrm{M}, 10^{-8} \mathrm{M}, 10^{-10} \mathrm{M}$ and $10^{-12} \mathrm{M}$ were prepared in distilled water to examine the sensitivity of the fabricated SERS substrates. The Raman spectra were measured using a Bruker Senterra Raman microscope. Since the highest SERS sensitivity in detecting R6G and MB molecules were obtained by employing laser excitation at $532 \mathrm{~nm}$, 18,19 all Raman measurements with the fabricated SERS substrates were carried out using a green laser at $532 \mathrm{~nm}$ with $2 \mathrm{~mW}$ of incident power. The laser beam diameter on the sample surface was about $1 \mu \mathrm{m}$ achieved using a 50X microscope objective. The spectral resolution of the spectrometer was set at $3 \mathrm{~cm}^{-}$ 1. All the Raman spectra were recorded with a data acquisition time of 2 seconds. The Raman spectra were background corrected. $2 \mu \mathrm{l}$ of the aqueous R6G or MB solution was directly spread on the SERS active area $(0.5 \mathrm{~cm} \times 0.5 \mathrm{~cm})$ and was dried under ambient condition.

\section{Results and discussion}

Figure 2 shows the Raman spectra of R6G and MB molecules adsorbed on the SERS substrates prepared from the analyte solutions at 10-8 M. The characteristic Raman bands of R6G at 615, 775, $1127,1182,1310,1362,1508,1573$, and $1649 \mathrm{~cm}^{-1}$ (Figure $2(1)^{13,18}$ and of MB at 495, 497, 677, 768, $890,1067,1181,1331,1396,1411,1544$, and $1620 \mathrm{~cm}^{-1}$ were measured (Figure 2(2) ${ }^{19}$ Raman spectra of R6G and MB prepared from the solutions with concentrations of $10^{-10} \mathrm{M}$ and $10^{-9} \mathrm{M}$, were recorded over nine random spots to evaluate the uniformity in sensitivity of the SERS substrates, respectively . As displayed in Figure 3, the R6G and MB Raman peaks were detected on all nine spots with similar peak intensities. The relative standard deviations (RSDs) were evaluated from the SERS spectra of the randomly selected nine spots for R6G and MB molecules and the RSD values for the main Raman peaks are summarized in Table 1. The averaged RSD values were determined to be about $15.93 \%$ and $5.86 \%$ for $\mathrm{R} 6 \mathrm{G}$ and $\mathrm{MB}$ respectively. The observation suggests that the analytes were uniformly distributed on the SERS substrates and the enhancement of the R6G and MB Raman signals with the substrates were highly reproducible over the SERS active area.

To determine the detection limits and the EFs of the fabricated SERS substrates, Raman spectra were measured using the SERS substrates and the silver nanoparticles coated silicon wafer (un-patterned) 
with the prepared R6G and MB solutions. Figure 4 shows the SERS spectra of R6G and MB. The silver nanoparticles coated wafer without the nanospikes was only able to detect R6G and MB molecules at a concentration of $10^{-5} \mathrm{M}$. Figure 4 (1) depicts the SERS spectra of R6G at $10^{-5}$ and $10^{-12} \mathrm{M}$. All the characteristic R6G Raman bands were observed even with a solution of $10^{-12} \mathrm{M}$ concentration for the fabricated substrate. This result indicates that the fabricated SERS substrates were able to sense about eight R6G molecules in the focal spot of the excitation laser beam assuming these molecules are spread uniformly over the substrate. Figure 4 (2) displays the SERS spectra of MB at $10^{-5}$ and $10^{-10} \mathrm{M}$. The EFs were estimated using the following expression:

where $\mathrm{I}_{S E R S}$ represents the SERS intensities of the Raman peaks from the adsorbed analyte molecules on the SERS active substrate; $\mathrm{I}_{\text {Ref }}$ represents the normal Raman signals from the adsorbed analyte molecules on the silver nanoparticles coated flat silicon substrate; $\mathrm{N}_{\text {SERS }}$ and $\mathrm{N}_{\text {Ref }}$ represent the corresponding number of analyte molecules excited by the laser beam. Assuming there is no specific binding between the analyte and the sample surface, the number of adsorbent molecules on the surface is proportional to the concentration of the analyte solution. ${ }^{20}$ Using the above equation and the assumptions, the EFs were determined to be about $3 \times 10^{7}, 4.3 \times 10^{7}, 5.3 \times 10^{7}, 7.7 \times 10^{7}$, and $1.03 \times 10^{8}$ for the corresponding Raman peaks at $615,775,1362,1508$ and $1649 \mathrm{~cm}^{-1}$, respectively. It is noted that a maximum EF approaching $10^{8}$ was achieved for the Raman band at $1649 \mathrm{~cm}^{-1}$. Similary for MB, the EFs for the main Raman peaks at 768, 1067, 1181, 1396 and $1620 \mathrm{~cm}^{-1}$ were, estimated to be, $1.3 \times 10^{5}$, $1.1 \times 10^{5}, 1.3 \times 10^{5}, 1.7 \times 10^{5}$, and $2.3 \times 10^{6}$, respectively. The measured high EFs clearly indicated that the silver nanoparticles (about $50 \mathrm{~nm}$ ) formed on the nanostructures significantly enhanced the local electromagnetic fields in the visible spectral region. In addition, higher resonance Raman enhancement due to strong absorption of R6G molecules from the $532 \mathrm{~nm}$ laser excitation led to the higher EFs as compared to those of the MB. The high sensitivity of the fabricated SERS substrates suggests that these substrates could be used as efficient SERS sensors for other analytes as well.

The reusability of the fabricated SERS substrates was examined using the R6G and MB solutions at concentrations of $10^{-10} \mathrm{M}$ and $10^{-9} \mathrm{M}$, respectively. After the Raman spectrum was taken, the substrates were cleaned by immersing in distilled water for $10 \mathrm{~min}$ to remove the adsorbed analytes and dried with nitrogen gas. Prior to re-adsorbing the analyte, the Raman spectrum of the cleaned substrate was recorded. Total 10 cycles of washing and re-adsorbing R6G and MB molecules on the SERS substrates 
were performed. No trace of R6G and MB was detected in the Raman spectra after washing, indicating the analyte molecules were completely rinsed off. Almost similar signal strengths for all the Raman bands were measured for re-adsorbed $\mathrm{R} 6 \mathrm{G}$ and $\mathrm{MB}$ on the same substrates for all the nine times, as compared to those of the analytes on the freshly fabricated substrates. Figure 5 shows the Raman spectra of R6G and MB measured with the freshly made substrates, the same substrates after washing, and at the fifth and tenth re-adsorptions. The result strongly demonstrated that the prepared SERS substrate is reusable. The reusability also suggests that the fabricated nanostructured SERS sensor would be costeffective. To the best of our knowledge, no clear demonstration of such reusability of other nanostructured SERS substrates has been reported.

\section{Conclusions}

In summary, we have developed highly sensitive and reusable SERS substrates. The SERS substrates can sense R6G and $\mathrm{MB}$ at concentrations down to $10^{-12} \mathrm{M}$ and 100 pico-molar, respectively. The sensitivity of the substrate is quite homogenous over the SERS active region. These sensors can be reused, allowing reliable and cost-effective means for sensitive detection of chemical. Further improvement of the sensitivity could be achieved by optimizing the coverage of silver particles on the silicon nanospikes. The developed SERS substrates in conjunction with portable Raman spectrometers would offer sensitive, reliable, and low-cost approaches to sense other chemicals and biological pathogens.

\section{Acknowledgements}

This work was partially supported by the Polymer-Based Materials for Harvesting Solar Energy, an Energy Frontier Research Center funded by the U.S. Department of Energy, Office of Science, Basic Energy Sciences (Award \#DE-SC0001087). This research was also supported by in part by an appointment to the Faculty Research Participation Program at the U.S. Army Natick Soldier Research, Development and Engineering Center (NSRDEC) administered by the Oak Ridge Institute for Science and Education through an interagency agreement between the U.S. Department of Energy and NSRDEC.

\section{References}


[1] P. Measor, L. Seballos, D. Yin, J.Z. Zhang, E.J. Lunt, A.R. Hawkins, H. Schmidt, On-chip surface-enhanced Raman scattering detection using integrated liquid-core waveguides, Appl. Phys. Lett. 90 (2007) 211107.

[2] K. Kneipp, Y. Wang, H. Kneipp, L.T. Perelman, I. Itzkan, R.R. Dasari, M.S. Feld, Single molecule detection using surface-enhanced Raman scattering (SERS), Phys. Rev. Lett. 78 (1997) 1667.

[3] R.J. Brown, M.J. Milton, Nanostructures and nanostructured substrates for surface - enhanced Raman scattering (SERS), J. Raman Spectrosc. 39 (2008) 1313-1326.

[4] D. Cialla, A. März, R. Böhme, F. Theil, K. Weber, M. Schmitt, J. Popp, Surface-enhanced Raman spectroscopy (SERS): progress and trends, Analytical and bioanalytical chemistry. 403 (2012) 27-54.

[5] L. Yang, J. Liu, Progress in multifunctional surface enhanced Raman spectroscopy substrate for detection, RSC Advances. 4 (2014) 49635-49646.

[6] X. Chen, C. Jiang, S. Yu, Nanostructured Materials for Applications in Surface Enhanced Raman Scattering, CrystEngComm.16 (2014) 9959-9973.

[7] Q. Fu, D. Zhang, Y. Chen, X. Wang, L. Han, L. Zhu, P. Wang, H. Ming, Surface enhanced Raman scattering arising from plasmonic interaction between silver nano-cubes and a silver grating, Appl. Phys. Lett. 103 (2013) 041122.

[8] H. Liu, L. Zhang, X. Lang, Y. Yamaguchi, H. Iwasaki, Y. Inouye, Q. Xue, M. Chen, Single molecule detection from a large-scale SERS-active Au79Ag21 substrate, Scientific reports. 1 (2011).

[9] W. Hoffmann, G. Verbeck, Toward a Reusable Surface-Enhanced Raman Spectroscopy (SERS) Substrate by Soft-Landing Ion Mobility, Appl. Spectrosc. 67 (2013) 656-660.

[10] X. Li, H. Hu, D. Li, Z. Shen, Q. Xiong, S. Li, H.J. Fan, Ordered array of gold semishells on TiO2 spheres: An ultrasensitive and recyclable SERS substrate, ACS applied materials \& interfaces. 4 (2012) 2180-2185.

[11] S.M. Mahurin, J. John, M.J. Sepaniak, S. Dai, A reusable surface-enhanced Raman scattering (SERS) substrate prepared by atomic layer deposition of alumina on a multi-layer gold and silver film, Appl. Spectrosc. 65 (2011) 417-422.

[12] G. Sinha, L.E. Depero, I. Alessandri, Recyclable SERS substrates based on Au-coated ZnO nanorods, ACS applied materials \& interfaces. 3 (2011) 2557-2563.

[13] H. Huo, C. Wang, H. Ren, M. Johnson, M. Shen, Surface enhanced Raman scattering sensing with nanostructures fabricated by soft nanolithography, Journal of Macromolecular Science ${ }^{\circledR}$, Part A: Pure and Applied Chemistry. 46 (2009) 1182-1184.

[14] E.D. Diebold, N.H. Mack, S.K. Doorn, E. Mazur, Femtosecond laser-nanostructured substrates for surface-enhanced Raman scattering, Langmuir. 25 (2009) 1790-1794.

[15] Y. Han, X. Lan, T. Wei, H. Tsai, H. Xiao, Surface enhanced Raman scattering silica substrate fast fabrication by femtosecond laser pulses, Applied Physics A. 97 (2009) 721-724.

[16] X. Lan, Y. Han, T. Wei, Y. Zhang, L. Jiang, H. Tsai, H. Xiao, Surface-enhanced Ramanscattering fiber probe fabricated by femtosecond laser, Opt. Lett. 34 (2009) 2285-2287.

[17] T. Siegfried, M. Kind, A. Terfort, O.J. Martin, M. Zharnikov, N. Ballav, H. Sigg, Reusable plasmonic substrates fabricated by interference lithography: a platform for systematic sensing studies, J. Raman Spectrosc. 44 (2013) 170-175.

[18] T. Vosgröne, A. Meixner, Surface-and Resonance-Enhanced Micro-Raman Spectroscopy of Xanthene Dyes: From the Ensemble to Single Molecules, ChemPhysChem. 6 (2005) 154-163.

[19] G. N. Xiao, S. Q. Man, Surface enhanced Raman scattering of methylene blue adsorbed on cap-shaped silver nanoparticles, Chem. Physics Lett. 447 (2007) 305-309. 
[20] R.P. Duyane, J.C. Hulteen, D.A. Treichel, Atomic force microscopy and surface enhanced Raman microsopy. I. Ag island films and Ag film over polymer nanosphere surfaces supported on glass, J. Chem. Phys. 99 (3) (1993) 2101-2115.

\section{Biographies}

Hardeep Singh Gill received his M.S. degree in physics from Punjabi University, Patiala in India. He is currently working towards Ph.D. degree with Prof. Kumar in the University of Massachusetts Lowell. He current research interests include optical spectroscopy, fabrication, and characterization of optoelectronic devices.

Sammaiah Thota received his M.S. degree in organic chemistry from Osmania University in India and $\mathrm{Ph} . \mathrm{D}$. degree in organic chemistry from the University of Massachusetts Lowell, MA, USA. He is currently with Prof. Kumar as a postdoctoral researcher at Center for Advanced Materials in the University of Massachusetts Lowell, MA, USA.

Lian Li received his Ph.D. degree in Physics from the Department of Physics, the University of Massachusetts Lowell, MA, USA. He is currently a visiting researcher with the U.S. Army Natick Soldier Research, Development and Engineering Center, Natick, MA. His current research interests include optical sensors, organic solar cells and nonlinear optics.

Haizhou Ren received his B.S. degree in physics from Xi'an Jiaotong University in China and is currently working towards Ph.D. degree at the University of Massachusetts Lowell.

Ravi Mosurkal received his Ph.D. degree in chemistry from University of Hyderabad, India in 1998. He did his postdoctoral work on nonlinear optical materials at the University of Durham, UK, before he joined late Prof. Tripathy's group at the University of Massachusetts Lowell in 2000 to work on dyesensitized solar cells. Since 2004, he has been an adjunct faculty at the University of Massachusetts Lowell and working on the development of environmentally safe flame retardant polymeric materials at U.S. Army Natick Soldier Research, Development and Engineering Center, Natick, MA. .

Jayant Kumar received his Ph.D. degree in Physics from Rutgers University, U.S.A. He is a professor in the Physics Department and director of Center for Advanced Materials at the University of Massachusetts Lowell. His research interests include optical sensors, nonlinear optics, organic solar cells, etc. 


\section{Figures}

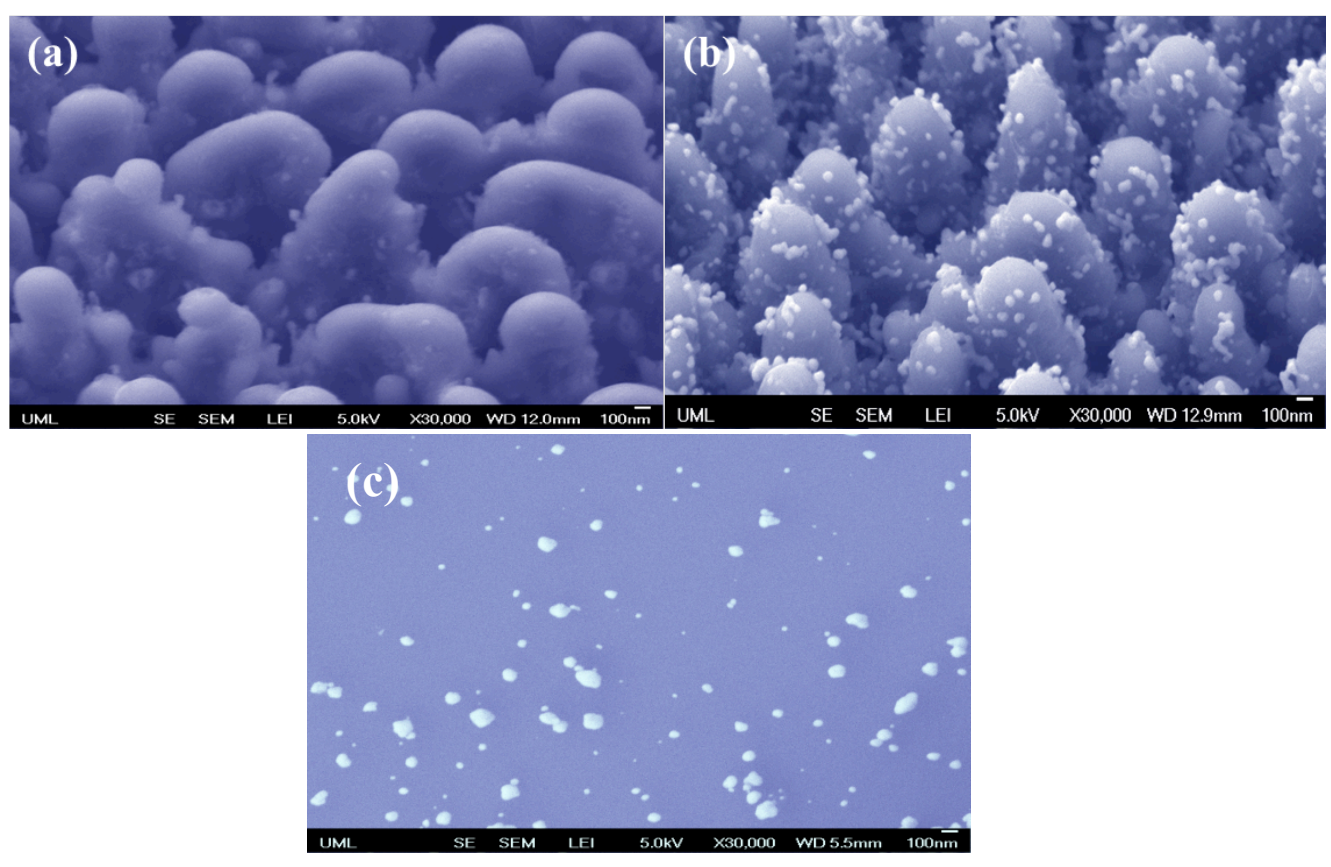

Figure 1. SEM images of (a) nanospikes formed on a silicon wafer by the FS laser pulses, (b) the nanospikes coated with silver nanoparticles and (c) the flat silicon surface coated with silver nanoparticles.
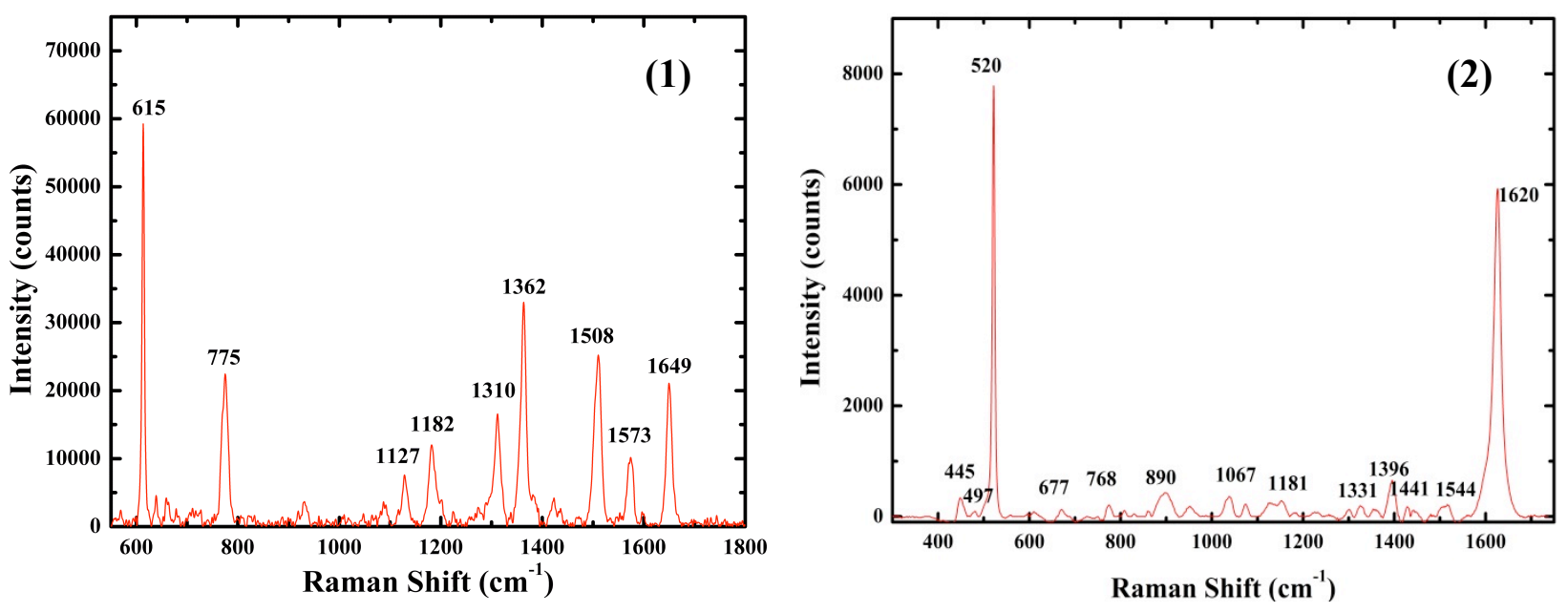

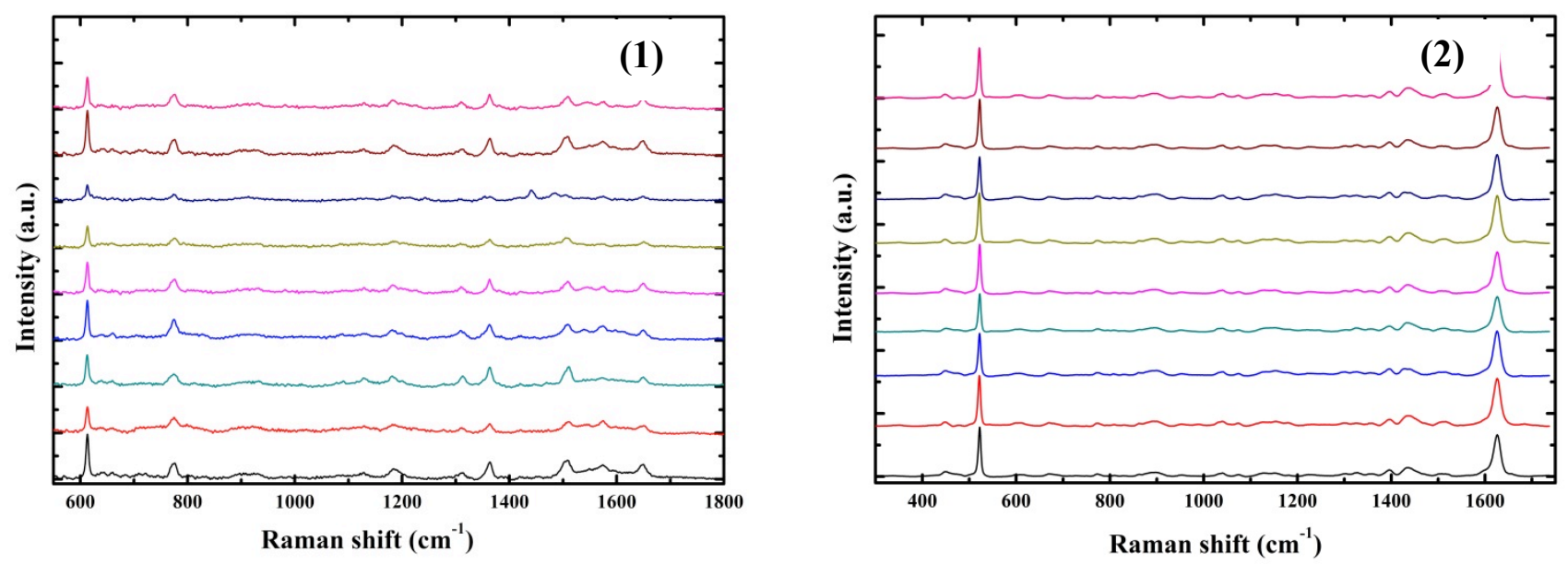

Figure 3. Raman spectra over nine randomly selected spots from (1) a R6G molecules adsorbed SERS substrate from a $10^{-10}$ $\mathrm{M}$ solution and (2) a MB molecules adsorbed substrate from a $10^{-9} \mathrm{M}$ solution.
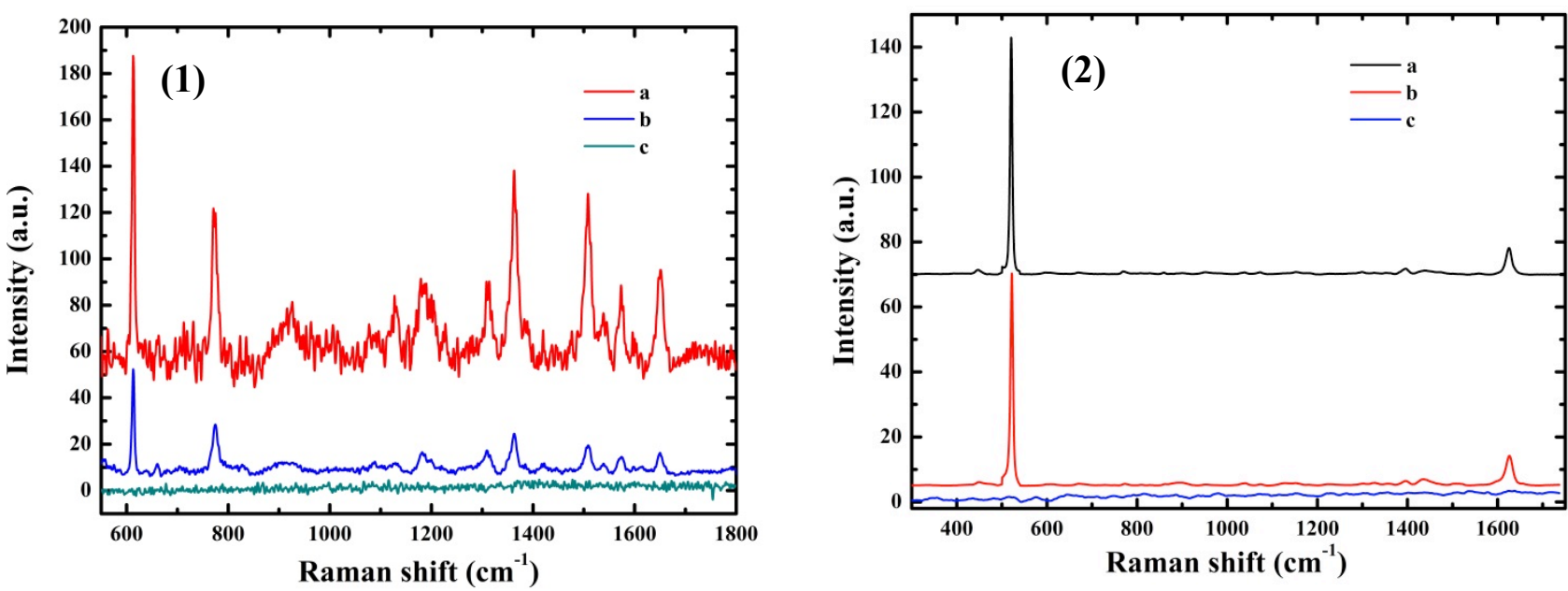

Figure 4. Raman spectra of (1) R6G molecules adsorbed on (a) a SERS substrate with a $10^{-12} \mathrm{M}$ solution, (b) a silver nanoparticles coated flat silicon surface using a solution at $10^{-5} \mathrm{M}$ and (c) a silver nanoparticles coated flat silicon surface using a solution at $10^{-12} \mathrm{M}$. (2) MB molecules absorbed on (a) a SERS substrate with a $10^{-10} \mathrm{M}$ solution, (b) a silver nanoparticles coated flat silicon surface using a solution at $10^{-5} \mathrm{M}$ and (c) a silver nanoparticles coated flat silicon surface using a solution at $10^{-10} \mathrm{M}$. 

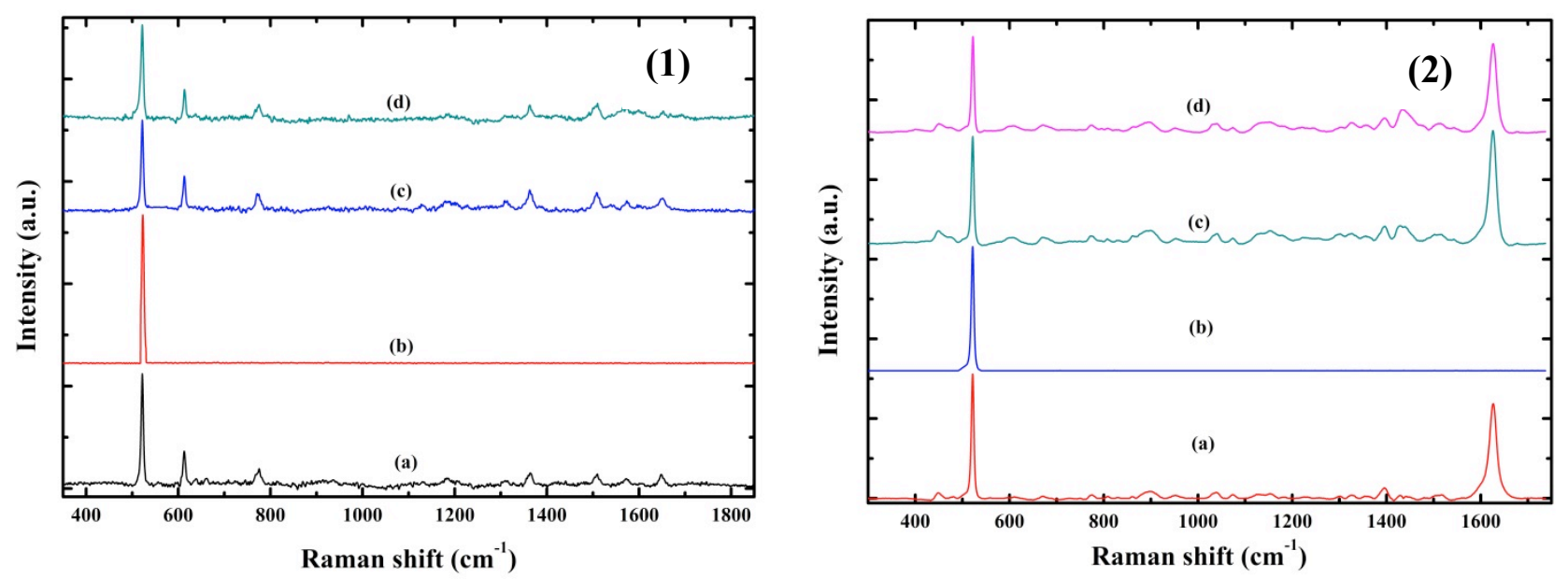

Table1: RSD values for the major peaks for the R6G and MB SERS spectrum.

Table1: Estimated RSD values of the main Raman peaks for R6G and MB molecules.

\begin{tabular}{cccccccccc}
\hline $\begin{array}{c}\text { Peak position } \\
\left(\mathbf{c m}^{-1}\right) \text { R6G }\end{array}$ & $\mathbf{6 1 5}$ & $\mathbf{7 7 5}$ & $\mathbf{1 1 2 7}$ & $\mathbf{1 1 8 2}$ & $\mathbf{1 3 1 0}$ & $\mathbf{1 3 6 2}$ & $\mathbf{1 5 0 8}$ & $\mathbf{1 5 7 3}$ & $\mathbf{1 6 4 9}$ \\
\hline RSD (\%) & 23.3 & 19.0 & 24.3 & 14.0 & 18.3 & 17.8 & 13.9 & 16.8 & 12.8 \\
$\begin{array}{c}\text { Peak position } \\
\left(\mathbf{c m}^{-1}\right) \mathbf{M B}\end{array}$ & $\mathbf{4 4 5}$ & $\mathbf{6 7 7}$ & $\mathbf{7 6 8}$ & $\mathbf{8 9 0}$ & $\mathbf{1 0 6 7}$ & $\mathbf{1 1 8 1}$ & $\mathbf{1 3 9 6}$ & $\mathbf{1 5 4 4}$ & $\mathbf{1 6 2 0}$ \\
RSD (\%) & 5.11 & 4.43 & 8.30 & 5.60 & 6.30 & 4.45 & 5.60 & 4.80 & 8.21 \\
\hline
\end{tabular}

